\title{
Construction of a primary DNA fingerprint database for cotton cultivars
}

\author{
Y.C. Zhang, M. Kuang, W.H. Yang, H.X. Xu, D.Y. Zhou, Y.Q. Wang, \\ X.A. Feng, C. Su and F. Wang \\ State Key Laboratory of Cotton Biology, \\ Cotton Research Institute of China Academy of Agricultural Science, \\ Anyang, Henan, China \\ Corresponding author: W.H. Yang \\ E-mail: cottontest@126.com
}

Genet. Mol. Res. 12 (2): 1897-1906 (2013)

Received February 6, 2012

Accepted September 20, 2012

Published January 30, 2013

DOI http://dx.doi.org/10.4238/2013.January.30.3

\begin{abstract}
Forty core primers were used to construct a DNA fingerprint database of 132 cotton species based on multiplex fluorescence detection technology. A high first successful ratio of $99.04 \%$ was demonstrated with tetraplex polymerase chain reaction. Forty primer pairs amplified a total of 262 genotypes among 132 species, with an average of 6.55 per primer and values of polymorphism information content varying from 0.340 to 0.882 . Conflicting DNA homozygous ratios were found in various species. The highest DNA homozygous ratio was found in landrace standard cultivars, which had an $81.46 \%$ DNA homozygous ratio. The lowest occurred in a group of 2010 leading cultivars with a homozygous ratio of $63.04 \%$. Genetic diversity of the 132 species was briefly analyzed using unweighted pair-group method with arithmetic means.
\end{abstract}

Key words: DNA fingerprint database; Simple sequence repeat; Fluorescence detection 


\section{INTRODUCTION}

Cotton is the leading natural fiber crop in the world as well as an important source of oil and seed meal (Rahman et al., 2002). The authorization and release of high-quality cultivars play an important role in agricultural production. During recent years, some significant breeding parents have been used frequently, resulting in genetic similarity of cotton cultivars and in difficulty for distinguishing them based on phenotypic performance. Transgenic technology has added to the challenge because modifying just a single genotype can produce a new cultivar. These characteristics have disordered the cotton market and added difficulty to the supervision of seed production and trade. Therefore, DNA fingerprint identification is of prime importance for ensuring robust quality seed (Ashok et al., 2011). The construction of a DNA fingerprint for cotton cultivars is essential. The International Union for the Protection of New Varieties of Plants (UPOV, 2005) recommends the use of simple sequence repeat (SSR) and single nucleotide polymorphism markers for the examination of crop distinctness, uniformity, and stability (DUS). Compared to single nucleotide polymorphisms, SSRs have been successfully used in the construction of DNA fingerprints, genetic diversity studies, and quantitative trait loci and are useful for a variety of applications in plant genetics. Because they are multiallelic and co-dominant and have Mendelian inheritance, SSRs are easy to score and are reproducible via polymerase chain reaction (PCR).

Microsatellite, or SSR, analysis is performed with PCR using defined oligonucleotide primers. Several methods have been adopted to analyze the length of PCR products. The most used technology is $6 \%$ polyacrylamide gel electrophoresis stained with silver nitrate (Creste et al., 2001). However, this method is time-consuming, especially when dealing with a large number of individuals. Fluorescent-labeled microsatellite primers detected through capillary electrophoresis have become high throughput and automated as a fluorescence-based SSR detection and allele sizing method performed on a DNA fragment analyzer. An advantage of fluorescence-labeled SSR genotyping is that several SSRs can be simultaneously separated in a single capillary or gel lane if the SSR fragments have non-overlapping sizes or are labeled with different colors. Thus, multiplex PCR combined with automated fluorescence detection can significantly increase the amount of information generated per assay and reduce labor costs associated with large-scale SSR genotyping. To date, this system has been used in maize (Wang et al., 2007), cotton (Liu et al., 2000), and soybean (Sayama et al., 2011).

DNA fingerprinting is a DNA-based identification system that depends on the genetic differences among individuals or organisms. The database can be used for cultivar identification, crossmonitoring, and intellectual property rights protection. Constructing a fingerprint of the cotton species would not only identify cotton species but also analyze their genetic distance and relationships with one another. Hou (2007) used 35 SSR markers to construct 96 silkworm race fingerprints and analyze genetic distance based on their origins. In this study, we constructed 132 DNA fingerprints of cotton species using a fluorescence-labeling detection system and analyzed the genetic diversity of the species. The results are expected to provide a basis for species identification and variety protection.

\section{MATERIAL AND METHODS}

\section{Material}

One hundred and thirty-two cotton cultivars including 8 Gossypium barbadense and 
124 G. hirsutum were used in this experiment (Table 1). The numbers marked with an asterisk in Table 1 are $G$. barbadense. The 132 species included 37 that were leading upland cotton cultivars in 2010, 52 source germplasms, 30 DUS standard cultivars, and 12 landrace standard cultivars. The 37 upland cultivars were collected from seed companies in China and covered a large cultivated area in 2010; they are coded 1 to 37 . The 30 DUS standard cultivars were once used for cotton DUS testing and are coded 38 to 67 . Source germplasms were considered lines or varieties from which 20 or more applied varieties have been derived, all of which were provided by Dr. X.M. Du and coded 68 to 120 . The 12 standard landrace cultivars were provided by authorized breeders and coded 121 to 132 .

Table 1. One hundred and thirty-two cotton species used in this experiment.

\begin{tabular}{|c|c|c|c|c|c|c|c|}
\hline Code & Species & Code & Species & Code & Species & Code & Species \\
\hline 1 & Hanmian 802 & 34 & Xinluzao 48 & 67 & Xuzhou 142 & 100 & Ganmian 1 \\
\hline 2 & Jichuangmian 1 & 35 & Xinluzao 42 & 68 & DPL 14A & 101 & Shanmian 3 \\
\hline 3 & Shikang 126 & 36 & Zhongmiansuo 41 & 69 & DPL 15 & 102 & Chaoyangmian 1 \\
\hline 4 & Guoxinmian 3 & 37 & Xinluzao 36 & 70 & Stoneville 4 & 103 & Jinmian 1 \\
\hline 5 & Lumianyan 30 & 38 & Sumian 15 & 71 & Stoneville 4B & 104 & Jinmian 2 \\
\hline 6 & Lumianyan 28 & 39 & Pengzeyahuang & 72 & Stoneville 2B & 105 & Keke 1543 \\
\hline 7 & Shannongshengmian 1 & 40 & Lingshiguozhi & 73 & Coker100 & 106 & Yishuhong \\
\hline 8 & Renhe 39 & 41 & Zhongmiansuo 19 & 74 & Delfos 531 & 107 & 31413 \\
\hline 9 & Yuza 37 & 42 & Zhongmiansuo 13 & 75 & Empire & 108 & Jimian 1 \\
\hline 10 & Zhongmiansuo 63 & 43 & Stoneville 825 & 76 & Foster 6 & 109 & Zhongmiansuo 12 \\
\hline 11 & Kaimian 5 & 44 & Eguangduanguozhi & 77 & Guannong 1 & 110 & Henshanmian 1 \\
\hline 12 & Jinke 178 & 45 & Zhongmiansuo 30 & 78 & Trice & 111 & Zhongmiansuo 10 \\
\hline 13 & Kemian 3 & 46 & Xinmian 33B & 79 & Jinyu 3 & 112 & Lambright GL-5 \\
\hline 14 & Siza 3 & 47 & Zhongmiansuo 12 & 80 & Jinyu 9 & 113 & Micnarie 210 \\
\hline 15 & Suza 3 & 48 & Chuanjian 1 & 81 & King & $114 *$ & Changrong 3 \\
\hline 16 & Fumian 2 & 49 & Xuzong 1 & 82 & Jiangsumian 1 & $115^{*}$ & Mcixxifl \\
\hline 17 & Ezamian 26 & 50 & 31413 & 83 & Jiangsumian 2 & $116^{*}$ & Xinhaimian \\
\hline 18 & Ezamian 17F1 & 51 & Zhongmiansuo 23 & 84 & Jiangsumian 3 & $117^{*}$ & $8763-И$ \\
\hline 19 & Ezamian 23F1 & 52 & Jimian 25 & 85 & $57-681$ & $118^{*}$ & C- 6022 \\
\hline 20 & Gangzamian 8F1 & 53 & Guokangmian 1 & 86 & Dongting 1 & $119^{*}$ & 9122-И \\
\hline 21 & Hanza $154 \mathrm{~F} 1$ & 54 & Ekangmian 9 & 87 & Gangyedaizimian & $120 *$ & Junhai 1 \\
\hline 22 & Daiza 1F1 & 55 & Xinluzao 1 & 88 & DPL 16 & 121 & Ezamian 10 \\
\hline 23 & Wanza 31 & 56 & Lumian 14 & 89 & Zhongmiansuo 2 & 122 & Suza 3 \\
\hline 24 & Wanza 5 & 57 & Yumian 19 & 90 & Zhongmiansuo 3 & 123 & Xiangzamian 10 \\
\hline 25 & Quanyin 2 & 58 & Siza 3 & 91 & Zhongmiansuo 4 & 124 & Zhongmiansuo 63 \\
\hline 26 & Guofengmian $12 \mathrm{~F} 1$ & 59 & Luxu 1 & 92 & Shanmian 4 & 125 & Zhongmiansuo 70 \\
\hline 27 & Ezamian 28F1 & 60 & Zhongmiansuo 16 & 93 & Shanmian 5 & 126 & Lumianyan 21 \\
\hline 28 & Xiangzamian 7 & 61 & Beiersinuo & 94 & Liaomian 3 & 127 & Lumianyan 28 \\
\hline 29 & Xiangnongmian 8 & 62 & Xuzhoubanbanmian & 95 & Xuzhou 209 & 128 & Zhongzhimian 2 \\
\hline 30 & Xinluzhong 33 & 63 & Liaomian 15 & 96 & Xuzhou 1818 & 129 & Zhongmiansuo 13 \\
\hline 31 & Xinluzhong 28 & 64 & $108 \mathrm{~F}$ & 97 & Uganda 4 & 130 & Zhongmiansuo 13 \\
\hline 32 & Xinluzhong 47 & 65 & Chuanmian 56 & 98 & Zhongmiansuo 7 & 131 & Xinluzao 33 \\
\hline 33 & Zhongmiansuo 41 & $66^{*}$ & Hai 7124 & 99 & $52-128$ & 132 & Xinluzao 42 \\
\hline
\end{tabular}

*Gossypium barbadense and the other materials are G. hirsutum.

\section{SSR molecular marker analysis}

Cotton genomic DNA was extracted from 3 individual dry seeds of each cultivar following a method described by Kuang (2010). The quality and quantity of DNA were estimated by measuring optical density at $260 / 280 \mathrm{~nm}$ with an ultraviolet spectrophotometer.

We carried out a primary survey among 12 standard landrace cultivars of which 40 primers with high polymorphism and even distribution throughout the genome ( 1 or 2 per chromosome) were selected as the core primers (Table 2). All of the primers were separated 
into 10 groups, and each group of tetraplex PCR combinations was labeled with a different fluorescence color (6-carboxyfluorescein, 6-carboxy-X-rhodamine, 5-hexachloro-fluorescein, tetramethyl-6-carboxyrhodamine). No overlap of amplification products of primers in the same group was allowed. All of the primers were synthesized by Microread Company in Beijing. The details of the microsatellite markers can be found in the Cotton Microsatellite Database (http://www.cottonmarker.org).

\begin{tabular}{|c|c|c|c|c|c|c|c|}
\hline Group & Primer & Map location & Fluorescence & Group & Primer & Map location & Fluorescence \\
\hline \multirow[t]{4}{*}{1} & BNL3442 & 11 & FAM & 6 & JESPR110 & 23 & FAM \\
\hline & NAU3254 & 1 & HEX & & NAU1200 & 5 & HEX \\
\hline & NAU3110 & 19 & TAMAR & & JESPR292 & 16 & TAMAR \\
\hline & MUCS101 & 4 & ROX & & BNL2960 & 10 & ROX \\
\hline \multirow[t]{4}{*}{2} & NAU934 & 5 & FAM & 7 & NAU1071 & 3 & FAM \\
\hline & NAU905 & $6 ; 25$ & HEX & & BNL1421 & 13 & HEX \\
\hline & NAU874 & 6 & TAMAR & & BNL3646 & 20 & TAMAR \\
\hline & NAU1028 & 17 & ROX & & NAU2277 & 2 & ROX \\
\hline \multirow[t]{4}{*}{3} & BNL3171 & 21 & FAM & 8 & NAU1167 & $17 ; 3$ & FAM \\
\hline & BNL830 & 15 & HEX & & CIR246 & 14 & HEX \\
\hline & BNL1231 & 11 & TAMAR & & BNL4030 & $22 ; 5$ & TAMAR \\
\hline & BNL2449 & $13 ; 10$ & ROX & & NAU1190 & 3 & ROX \\
\hline \multirow[t]{4}{*}{4} & CIR216 & 18 & FAM & 9 & CIR170 & 26 & FAM \\
\hline & NAU1125 & 24 & HEX & & BNL3261 & 12 & HEX \\
\hline & NAU859 & 9 & TAMAR & & NAU1369 & 8 & TAMAR \\
\hline & NAU3588 & 25 & ROX & & NAU1362 & 7 & ROX \\
\hline \multirow[t]{4}{*}{5} & BNL3140 & 23 & FAM & 10 & NAU3778 & 12 & FAM \\
\hline & NAU1103 & 21 & HEX & & DPL0442 & 20 & HEX \\
\hline & BNL1317 & $9 ; 23$ & TAMAR & & NAU2437 & 15 & TAMAR \\
\hline & BNL827 & 25 & ROX & & NAU5099 & 22 & ROX \\
\hline
\end{tabular}

FAM = 6-carboxyfluorescein; HEX = 5-hexachloro-fluorescein; TAMAR = tetramethyl-6-carboxyrhodamine; $\mathrm{ROX}=6$-carboxy-X-rhodamine.

PCR was performed in a total volume of $20 \mu \mathrm{L}$ containing $2 \mu \mathrm{L} 20 \mathrm{ng} / \mu \mathrm{L}$ genomic DNA, $1 \mu \mathrm{L} 5 \mathrm{mM}$ primer mixture, $8 \mu \mathrm{L} 2.5 \mathrm{X}$ buffer $\mathrm{V}$ (including deoxyribonucleotide triphosphate), $0.16 \mu \mathrm{L}$ Taq DNA polymerase, and double-distilled water. Amplification using tetraplex PCR was performed under the following conditions: a cycle of $4 \mathrm{~min}$ at $94^{\circ} \mathrm{C}$ for strand separation followed by 32 cycles of $45 \mathrm{~s}$ at $94^{\circ} \mathrm{C}$ for denaturation, $45 \mathrm{~s}$ at $55^{\circ} \mathrm{C}$ for annealing, and $45 \mathrm{~s}$ at $72^{\circ} \mathrm{C}$ for primer extension. A cycle followed at $60^{\circ} \mathrm{C}$ for $30 \mathrm{~min}$ and a hold at $15^{\circ} \mathrm{C}$ until removal.

The electrophoresis and visualization of the $\mathrm{PCR}$ products were performed on an $\mathrm{ABI} 3730$ DNA analyzer (Applied Biosystems, Foster City, CA, USA) using the GeneMapper software through measurement of the fluorescence intensity for each marker. Figure 1 presents the detection results of species by four fluorescent multiplex PCR sets in capillary electrophoresis. The sample prepared for sequencing was based on $1-\mu \mathrm{L}$ PCR products and $9 \mu \mathrm{L}$ gel loading buffer including $8.5 \mu \mathrm{L}$ deionized formamide and $0.5 \mu \mathrm{L} \mathrm{Liz-500} \mathrm{size} \mathrm{standard} \mathrm{(Applied} \mathrm{Biosystems).} \mathrm{The}$ number of alleles per locus as well as the combination type of polymorphic bands were recorded as different genotypes. The genetic diversity of each SSR locus was obtained by calculating the frequency of the genotype based on polymorphism information content (PIC) using the equation $\mathrm{PIC}_{\mathrm{i}}=1-\sum \mathrm{Plj}^{2}$, where $P l j$ is the frequency of the $l^{\text {th }}$ allele for locus $j$ and is summed over its $L$ alleles. A similarity matrix was calculated using Nei's genetic distance, and the general cluster of 132 species was analyzed with the unweighted pair-group method with arithmetic mean software. 


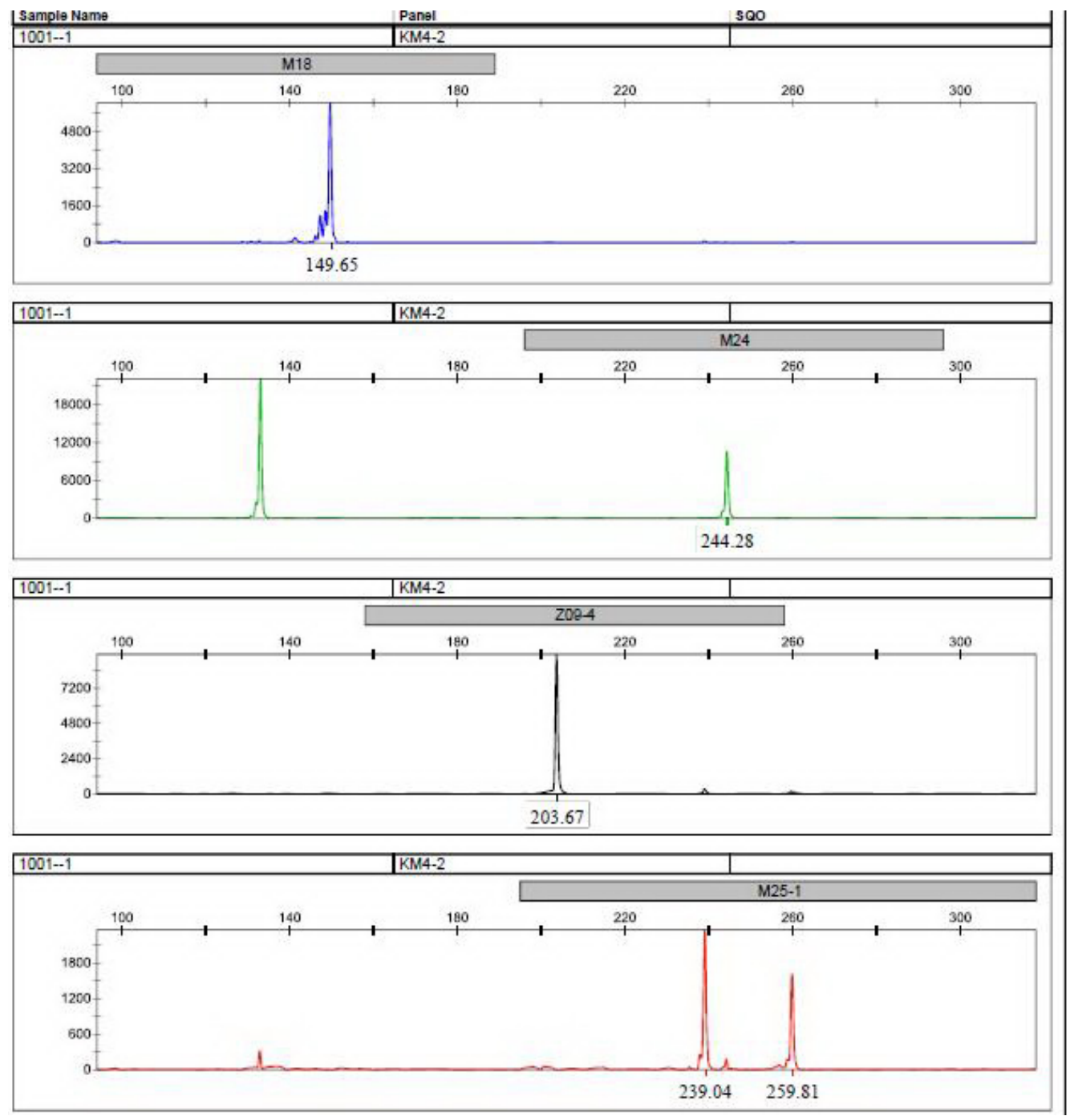

Figure 1. Hanmian 802 SSR locus genotypes amplified by Group 4.

\section{RESULTS}

\section{Microsatellite allelic diversity}

Forty primers divided into 10 groups were used to amplify 132 species; each species assayed 3 individuals. Frequency distribution analysis showed that $99.04 \%$ of the SSRs present in the tetraplex PCR were successfully amplified during the first test. Two individuals (codes 61-1 and 117-2) gave no or very little amplification despite being detected twice, and we suspect that the genomic DNA may have denatured before the test. Forty primers produced a total of 177 
fragments; 166 were polymorphic among 132 species, with $94.92 \%$ polymorphism. The size of the amplified fragment was 101-311 bp. The number of amplification genotypes generated by the 40 primers was 262. A maximum of 22 genotypes was amplified with primer BNL 1317, and a minimum of 3 genotypes was amplified with primers NAU5099, CIR170, BNL3140, BNL3646, and BNL830, with a mean of 6.55 genotypes per primer. The PIC value calculated to estimate the informativeness of each primer varied from 0.340 to 0.882 , with an average of 0.555 .

\section{DNA homozygous ratio and DNA fingerprint}

Three individuals were selected for each species, but not all of the individuals of the same species amplified the same genotype. We were not surprised by this result, as breeders have not selected the specific SSR loci, and conflicting DNA homozygous ratios from different sources of species can be expected. The highest DNA homozygous ratio (Table 3) was found in the landrace standard cultivars $(81.46 \%$ ); the lowest (63.04\%) occurred in the 2010 leading cultivars. That is because the landrace standard cultivars have come from authorized breeders, whereas the leading cultivars were obtained from seed companies. Three genotypes existed among the same species, and this circumstance can exist even in the seeds from authorized breeders. A potential explanation for the phenomenon is a residual number of heterotypic genotypes. For breeding, selection is based primarily on phenotypic traits; therefore, the markers in the important gene-coding regions of the genome and nearby locations are fixed quickly for the respective alleles, but the remaining markers are fixed at random and, as a result, show different phenotypes (Liu et al., 2007).

\begin{tabular}{|c|c|c|c|c|c|}
\hline Source & $3: 0$ & $2: 1$ & $1: 1: 1$ & $1: 1$ & $2: 0$ \\
\hline 2010 leading cultivars & 0.6304 & 0.3054 & 0.0601 & 0.0007 & 0.0034 \\
\hline DUS standard cultivar & 0.7458 & 0.1958 & 0.0250 & 0.0108 & 0.0225 \\
\hline Source germplasms & 0.6953 & 0.2538 & 0.0321 & & 0.0189 \\
\hline Landrace standard cultivars & 0.8146 & 0.1688 & 0.0167 & & \\
\hline
\end{tabular}

DUS = distinctness, uniformity, and stability.

Nevertheless, we found the highest heterozygous ratio in the 2010 leading cultivars (6.01\% for $1: 1: 1$ and $0.07 \%$ for $1: 1)$. The next highest were the source germplasms, DUS standard cultivars, and landrace standard cultivars with ratios of $3.21,2.5$, and $1.67 \%$ for $1: 1: 1$, respectively. Therefore, we propose that the analysis of 3 samples may be insufficient to display the distinctness of the seed, and deploying more than 3 samples for each individual may be preferable. We consider loci that appear in at least 2 individuals with the same genotype to be the true loci for the species. With the 40 SSR loci, we constructed a DNA fingerprint of the 132 species using a question mark to denote uncertainty. Table 4 shows the fingerprint of Fumian 2.

\section{Genetic distance and diversity}

The SSR amplification results were used to generate a genetic similarity matrix. Genetic similarity coefficients among 132 species ranged from 0.3202 to 0.9649 , with an average of 0.7902 , indicating that the species we used had a vast genetic base (Figure 2). The minimum co- 


\begin{tabular}{|c|c|c|c|c|c|c|c|c|c|c|}
\hline Primer & Size 1 & Size 2 & Size 3 & Size 4 & Size 5 & Primer & Size 1 & Size 2 & Size 3 & Size 4 \\
\hline M01 & 276 & 285 & 291 & & & M16 & 174 & 179 & & \\
\hline M02 & & & & 143 & & M17 & $?$ & & & \\
\hline M03 & 218 & & 228 & & & M17-1 & $?$ & & & \\
\hline $03-1$ & $?$ & & & & & M18 & & 150 & & \\
\hline M04 & 154 & & 166 & & & M19 & 214 & 223 & & \\
\hline M05 & ? & & & & & M20 & & 159 & & \\
\hline $05-1$ & $?$ & & & & & M21 & $?$ & & & \\
\hline M06 & 148 & & & 179 & & M21-1 & $?$ & & & \\
\hline $06-1$ & & & 161 & 167 & & M22 & $?$ & & & \\
\hline M07 & ? & & & & & M23 & ? & & & \\
\hline M09 & ? & & & & & M23-1 & & 186 & & 222 \\
\hline M10 & & 156 & & & & M24 & ? & & & \\
\hline M11 & & & 202 & & & M25 & 250 & & 269 & \\
\hline M11-1 & $?$ & & & & & $25-1$ & $?$ & & & \\
\hline M12 & $?$ & & & & & M26 & $?$ & & & \\
\hline M13 & 190 & & & & & Z08-1 & 241 & 247 & & \\
\hline M13-1 & & & 165 & & & Z09-4 & $?$ & & & \\
\hline M14 & $?$ & & & & & Z12-2 & 223 & 227 & & \\
\hline M15 & & 232 & & & 244 & Z20-1 & & 148 & & \\
\hline $15-1$ & & 110 & & & & Z22-2 & & 221 & & 228 \\
\hline
\end{tabular}

$?=$ stand for 1:1:1 genotype, which means that 3 individuals of the same species have 3 different genotypes.

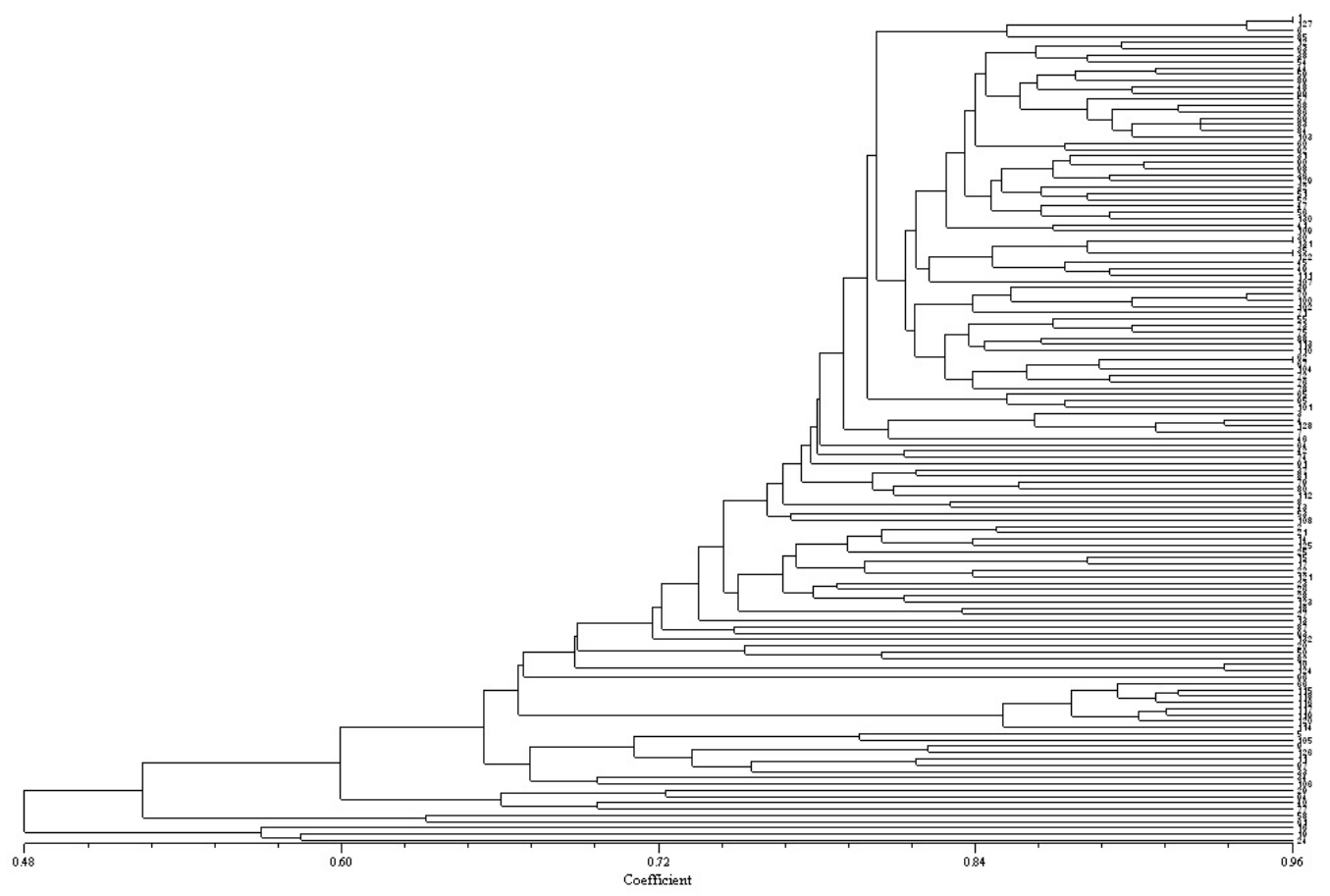

Figure 2. UPGMA dendrogram of 132 species based on SSR data.

efficient occurred between Simian 3 and Ezamian 23, indicating that these species were the least genetically similar. Four pairs of species had the maximum similarity. Four species had different 
origins but shared the same name: Lumianyan 28, Zhongmiansuo 63, Suza 3, and Xinluzao 42. These species belonged to the 2010 leading cultivars and landrace standard cultivars. Strikingly, 2 independent sources of Suza 3 and Xinluzao 42 from the 2010 leading cultivars were amplified by the 40 SSR loci, but they differed greatly from the seed provided by authorized breeders, displaying a similarity of 70.18 and $73.25 \%$, respectively. This result suggested a high probability that the seed company had changed the seed name for profit. Lumianyan 28 and Zhongmiansuo 63 had a similarity of 94.3 and $93.9 \%$ with the landrace standard cultivars, respectively. Zhongmiansuo 41 (codes 33 and 36) were 2010 leading cultivars from different companies with a similarity of $76.32 \%$. Among the 132 species, Fumian 2 was excellent, as its average genetic similarity to other species was 0.4457 , showing a large variability in genomic constitution. The general cluster of 132 species was analyzed with the unweighted pair-group method with arithmetic means based on similarity coefficients. Eight $G$. barbadense were clustered in the same group, and the species bred by the same institute belonged to the same group - for example, the Zhongmiansuo group, with the exception of Zhongmiansuo 41 (code 33). Zhongmiansuo 41 has been shown to originate from Zhongmiansuo 23 introgressed with an anti-insect gene. Therefore, we infer that code 33 maybe the false genotype of Zhongmiansuo 41.

\section{DISCUSSION}

The protection of new plant varieties in China commenced in 1997, and cotton has the fifth largest number of protected plant varieties after rice, maize, wheat, and soybean. More and more attention has been drawn to DNA fingerprinting as a means of protecting new varieties (Liu et al., 2007). Selecting a set of core primers is the first and pivotal step in establishing DNA fingerprints (Liu et al., 2010). In the present study, we selected 40 primers based on criteria that included high polymorphism, high amplification, and multiplex PCR amplification potential (George et al., 2004). These primers discriminated all 132 species, even those that shared the same name, and for the tetraplex primer combinations, only 1 PCR amplification and 1 capillary electrophoresis were required, resulting in a high success rate of $99.04 \%$. Our result suggests that applying the 40 core primers obtained in the present study may be a priority in tetraploid species.

Constructing a crop DNA fingerprint is imperative for ensuring agronomic performance and protecting intellectual rights. Intra-varietal heterogeneity exists extensively in cotton as an allotetraploid species. The results of this study indicate that the analysis of multiple individuals per species could provide an effective way of determining the essential genotype of a species. In the present study, we analyzed 3 individuals of each species from 4 kinds of source seeds. The existence of DNA heterozygous ratios led to a large amount of uncertain data, and further analysis is needed to determine the optimal number of individuals required to represent the true genotype of a species. Four sources of cotton seeds were used to construct the fingerprint as a result the seeds from authorized breeders amplified the highest homozygous ratio and the company seeds were the lowest. However, even the authorized seeds amplified heterozygous loci among the 3 individuals. This phenomenon may be explained by the within-variety variation owing to residual heterozygosity. However, residual heterozygosity may not account for the coexistence of both high heterozygous ratios and low homozygous ratios in the company seed. This result may be caused by the pursuit of commercial profit, as some cultivars may have intentionally been given different names or breed generation may 
have been reduced. For example, Zhongmiansuo 41 (codes 33 and 36), 2 cultivars with the same name, were largely different. The cluster analysis showed that code 33 was highly likely to belong to the false genotype, so authenticating cotton species during database construction is important. Establishing DNA fingerprints for crop varieties is a powerful means of normalizing the administration and management of these varieties. The SSR-based identification of rice varieties has become an official test in national trials in China (Ying et al., 2007). Considering the complexity of cotton, many problems remain to be resolved.

A general genetic diversity was analyzed in this study. Cultivars bred from the same institute were clustered into the same group, conforming with the results of previous research (Zhang et al., 2011). However, a vast genetic base was found that differed considerably from that uncovered through research using random-amplified polymorphic DNA and SSRs, in which the genetic diversity was narrow. Mehboo-ur-Rahman (2008) analyzed genetic diversity among 30 cultivars of $G$. arboreum based on random-amplified polymorphic DNA markers and revealed a limited diversity base. Sun et al. (2009) analyzed 61 colored-cotton lines using SSR markers and showed that the genetic background of colored cotton with elite properties was narrow. These discordant results may derive from differences in the materials used. The 132 species in this study covered mostly parental lines (source germplasms) applied to derive cultivars and some G. barbadense. Chen and Du (2006) analyzed 47 source germplasms and indicated that the genetic diversity was rich. Generally, pressure for high productivity and quality narrow genetic diversity. The results of this study provide data for variety improvement and the updating of diversity of germplasms as well as important methods and technologies for the construction of DNA fingerprints.

\section{ACKNOWLEDGMENTS}

The authors thank Dr. X.M. Du of the Cotton Research Institute and the Chinese Academy of Agricultural Sciences for providing materials.

\section{REFERENCES}

Ashok BD, Mamta PR, Manoj RB and Kshanada JM (2011). Identification and genetic purity testing of cotton $\mathrm{F}_{1}$ hybrid using molecular markers. India J. Biotechnol. 10: 301-306.

Chen G and Du XM (2006). Genetic diversity of source germplasm of Upland cotton in China as determined by SSR marker analysis. Yi Chuan Xue Bao 33: 733-745.

Creste S, Tulmann NA and Figueira A (2001). Detection of single sequence repeat polymorphisms in denaturing polyacrylamide sequencing gels by silver staining. Plant Mol. Biol. Rep. 19: 299-306.

George ML, Regalado E, Li W, Cao M, et al. (2004). Molecular characterization of Asian maize inbred lines by multiple laboratories. Theor. Appl. Genet. 109: 80-91.

Hou CX, Li MW, Zhang YH, Qian HY, et al. (2007). Analysis of SSR fingerprints in introduced silkworm germplasm resources. Agric. Sci. China 6: 620-627.

Kuang M, Yang WH, Xu HX and Wang YQ (2010). A rapid method of DNA extraction from single cotton seed. Mol. Plant Breed. 8: 827-831.

Liu LW, Yan W, Gong YQ, Zhao TM, et al. (2007). Assessment of genetic purity of tomato (Lycopersicon esculentum L.) hybrid using molecular markers. Sci. Horticulturae 115: 7-12.

Liu S, Saha S, Stelly D, Burr B, et al. (2000). Chromosomal assignment of microsatellite loci in cotton. J. Hered. 91: 326-332.

Liu XL, Ma L, Chen XK, Ying XM, et al. (2010). Establishment of DNA fingerprint identity for sugarcane cultivars in Yunnan, China. Acta Agron. Sin. 36: 202-210. 
Mehboo-ur-Rahman, Tahira Y, Nabila T, Ihsan U, et al. (2008). Studying the extent of genetic diversity among Gossypium arboreum L. genotypes/cultivars using DNA fingerprinting. Genet. Resour. Crop Evol. 55: 331-339.

Rahman M, Hussain D and Zafar Y (2002). Estimation of genetic divergence among elite cotton cultivars genotypes by DNA fingerprinting technology. Crop Sci. 42: 2137-2144.

Sayama T, Hwang TY, Komatsu K, Takada Y, et al. (2011). Development and application of a whole-genome simple sequence repeat panel for high-throughput genotyping in soybean. DNA Res. 18: 107-115.

Sun DL, Sun JL, Jia YH, Ma ZY, et al. (2009). Genetic diversiry of colored analyzed by simple sequence repeat markers. Int. J. Plant Sci. 170: 76-82.

UPOV (Union for the Protection of New Varieties of Plants) (2005). Guidelines for Molecular Marker Selection and Database Construction, BMT Guidelines (Proj. 3). UPOV, Geneva.

Wang FG, Zhao JR, Dai JR, Yi HM, et al. (2007). Selection and development of representative simple sequence repeat primers and multiplex SSR sets for high throughput automated genotyping in maize. Chin. Sci. Bull. 52: 215-223.

Ying JZ, Shi YF, E ZG, Zeng RZ, et al. (2007). Construction and testing of a primary microsatellite database of major rice varieties in China. Rice Sci. 14: 247-255.

Zhang Y, Wang XF, Li ZK, Zhang GY, et al. (2011). Assessing genetic diversity of cotton cultivars using genomic and newly developed expressed sequence tag-derived microsatellite markers. Genet. Mol. Res. 10: 1462-1470. 